Oikos 121: 1985-1994, 2012

doi: $10.1111 / j .1600-0706.2012 .20185 . x$

(C) 2012 The Authors. Oikos (C) 2012 Nordic Society Oikos

Subject Editor: Lars-Anders Hansson. Accepted 3 February 2012

\title{
Determining trophic niche width: an experimental test of the stable isotope approach
}

\author{
Patrick Fink, Elke S. Reichwaldt, Chris Harrod and Axel G. Rossberg \\ P. Fink (fink@limno.net), E. S. Reichwaldt and C. Harrod, Max-Planck-Inst. for Limnology, Dept for Physiological Ecology, August-Thienemann- \\ Strasse 2, DE-24306 Plön, Germany. Present address for PF: Univ. of Cologne, Cologne Biocenter, Zülpicher Strasse 47b, DE-50674 Köln, \\ Germany. Present address for ESR: The Univ. of Western Australia, School of Environmental Systems Engineering, M015, 35 Stirling Highway, \\ Crawley WA 6009, Australia. Present address for A. G. Rossberg: Queen's Univ. of Belfast, School of Biological Sciences, Belfast, UK. \\ Present address for CH: Univ. de Antofagasta, Inst. de Investigaciones Oceanológicas, Av. Angamos 601, Antofagasta, Chile.
}

\begin{abstract}
Determining the trophic niche width of an animal population and the relative degree to which a generalist population consists of dietary specialists are long-standing problems of ecology. It has been proposed that the variance of stable isotope values in consumer tissues could be used to quantify trophic niche width of consumer populations. However, this promising idea has not yet been rigorously tested. By conducting controlled laboratory experiments using model consumer populations (Daphnia sp., Crustacea) with controlled diets, we investigated the effect of individual-and population-level specialisation and generalism on consumer $\delta^{13} \mathrm{C}$ mean and variance values. While our experimental data follow general expectations, we extend current qualitative models to quantitative predictions of the dependence of isotopic variance on dietary correlation time, a measure for the typical time over which a consumer changes its diet. This quantitative approach allows us to pinpoint possible procedural pitfalls and critical sources of measurement uncertainty. Our results show that the stable isotope approach represents a powerful method for estimating trophic niche widths, especially when taking the quantitative concept of dietary correlation time into account.
\end{abstract}

Trophic niche width is typically measured as the diversity of resource types consumed by a consumer (McDonald 2002). It allows researchers to address questions on the ecology and evolution of various kinds of organisms in their ecological interactions with predators, prey and competitors (Van Valen 1965, Roughgarden 1972, Pigeon et al. 1997). Conventional diet analysis, which is typically conducted through gut content analysis (Hyslop 1980), is nowadays commonly used in conjunction with measurements of consumer stable isotope ratios. While the former provides more detailed information, the latter has the advantages of methodological simplicity and of representing the integrated assimilated diet of the consumer rather than just recent intake. However, consumer isotopic variance cannot be used as an indicator of individual specialisation without considering the relative isotopic variation in the available prey, as highlighted by Matthews and Mazumder (2004). Bearhop et al. (2004) proposed a widely-cited approach for the determination of the trophic (feeding) niche width of a consumer population by using the variance of the consumers' stable isotope $\left(\delta^{13} \mathrm{C}\right.$ and/or $\left.\delta^{15} \mathrm{~N}\right)$ ratios among individuals. In general, stable isotope analysis is a useful method for understanding the trophic ecology of consumers as the stable isotope ratios in their tissues reflect those of their diet in a generally predictable manner (Peterson and Fry 1987, Hobson and Clark 1992); there is a mean increase in $\delta^{13} \mathrm{C}$ by ca $1 \%$ and in $\delta^{15} \mathrm{~N}$ by ca $3.4 \%$ per trophic level due to trophic fractionation (Cabana and Rasmussen 1994, McCutchan et al. 2003). Therefore, if individuals are specialised on one food type only they will display isotopic values from this food type (adjusted for trophic fractionation), while individuals that feed on and incorporate many different food items with different isotopic values will have a mean isotopic value which reflects the different isotopic contributions in the diet (including fractionation). Reflecting observations from empirical data, this has been suggested multiple times in the literature (Fry et al. 1978, Bolnick et al. 2003, Matthews and Mazumder 2004). From this, Bearhop et al. (2004) predicted differences in the distribution of individual isotopic values for dietary specialists (SP; Fig. 1), and for two types of dietary generalists; generalist populations composed of individuals that all consume a wide range of resources (type A generalists, GA; Fig. 1) and generalist populations composed of individuals that are themselves specialised on different, narrow resource ranges (type B generalists, GB; Fig. 1). Distinguishing empirically between GA and GB by using traditional methods of diet analysis is extremely challenging (Van Valen 1965, Bearhop et al. 2004). The isotopic approach proposed by Bearhop et al. (2004) is an attractive alternative. Bearhop et al. (2004) further highlighted the use of consumer stable isotope values to estimate trophic niche width, as it yields 


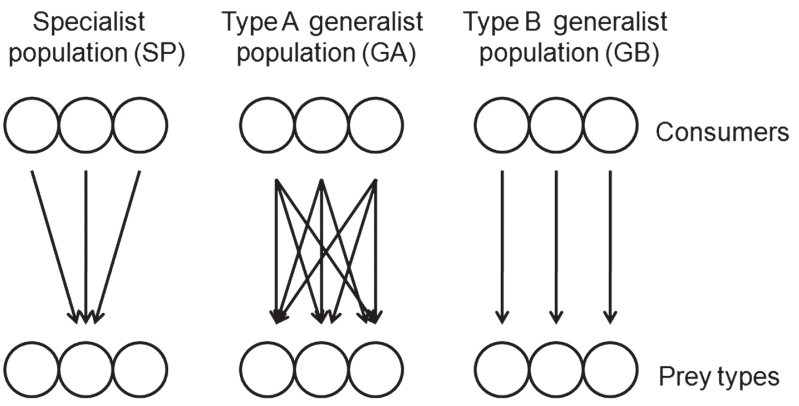

Figure 1. Distinction between specialist populations (SP), type A generalist populations composed of generalist individuals (GA), and type $\mathrm{B}$ generalist populations composed of specialist individuals (GB); classification after Bearhop et al. (2004).

a single measure on a continuous axis common to all species and provides a temporal integration of prey assimilation. Furthermore, the time frame of the temporal integration can be adjusted by the choice of the sampled consumer tissue, as the tissue's isotopic value reflects the diet that was assimilated during the period of tissue synthesis. For example, in vertebrate consumers muscles or bones integrate diets over longer times than blood or liver (Hobson 1999).

The distinction between type A and type B generalists can be seen as two extremes in a range of temporal continuity in resource usage. True type A generalist individuals in the sense of Bearhop et al. (2004) continuously switch between alternative diets, while a true type B generalist individual never switches, but constantly consumes the diet it is specialised on (Newsome et al. 2009, Vander Zanden et al. 2010). We propose that continuity in resource usage can be quantified in terms of the 'dietary correlation time', i.e. the typical length of time after which a consumer changes its diet. To translate the classification of Bearhop et al. (2004) into this context, we here consider type B generalists as those species where the dietary correlation time of individuals is of the order of magnitude of the natural life-time, or of the duration of the specific life stage considered in an analysis. In contrast, type A generalists frequently vary their diet over the full spectrum of available resources during their life-time or the life-stage considered (Fig. 2). The isotopic pattern found for type A generalists will depend on the relative magnitude of the dietary correlation time and the isotopic integration time of the tissue analyzed. Empirically, one can therefore distinguish between two kinds of type A generalists, $\mathrm{GA}_{\text {short }}$ and $\mathrm{GA}_{\text {long }}$ corresponding to tissue integration times that are short and long, respectively, compared to dietary correlation times of the consumers. As indicated in Fig. 2, shorter and longer tissue integration times for type A generalists will have similar effects on the isotope values as longer and shorter dietary correlation times, respectively. In this view, expressed also by Bearhop et al. (2004), the suffixes of $\mathrm{GA}_{\text {short }}$ and $\mathrm{GA}_{\text {long }}$ refer to tissue integration times rather than dietary correlation time. Type B generalists correspond to species that appear as $\mathrm{GA}_{\text {short }}$ even when tissues with integration times spanning the full life time of individuals are analyzed.

Obviously, these types (GA and GB) are, in their 'pure' forms, idealizations. Differences between individuals in

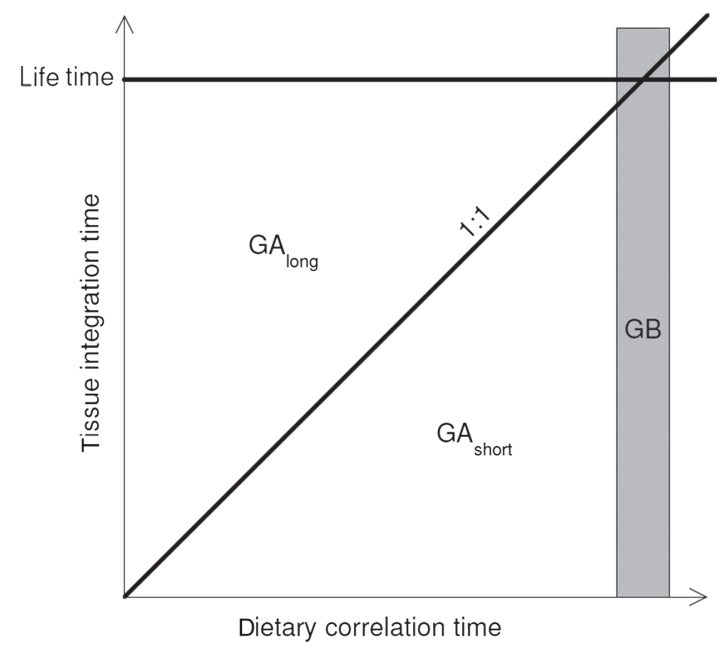

Figure 2. Schematic illustration of the distinctions between $\mathrm{GA}_{\text {short }}, \mathrm{GA}_{\text {long }}$ and GB. GA $\mathrm{A}_{\text {short }}$ and $\mathrm{GA}_{\text {long }}$ distinguish situations where tissue integration time is short or long compared to the dietary correlating time. GB describes generalist populations where dietary correlation time is of the order of magnitude of the natural life time of individuals.

phenotype, experience, and location are likely to lead to some dietary variation among individuals in most cases. This is what we intend to capture by introducing the notion of dietary correlation time (DCT) and speaking of a continuum of different types spanned by the extreme (meaning idealized) cases GA and GB. Therefore, researchers need to understand how precisely dietary correlation time would influence the variance of isotope values within a population in the field in order to reliably interpret stable isotope data from natural populations. Here we propose a model for the dependence of isotopic variance on dietary correlation time. The model predicts the isotopic variance of a population from dietary correlation time and populationlevel diet width.

While the predictions by Bearhop et al. (2004) and the suggestions they derived for using isotopic variance as a direct measure of trophic niche width have found wide application in recent years (Syväranta and Jones 2008, Martinez del Rio et al. 2009), the underlying ideas have not been subjected to controlled experimental tests, and the interpretation of results based on them remain largely qualitative. To experimentally address the effects of dietary correlation time and consumer specialisation or generalisation on the isotopic variance of a consumer population, we performed controlled laboratory experiments using the freshwater crustacean zooplankton Daphnia pulicaria as the consumer, and the green alga Scenedesmus obliquus (Chlorophyceae) as the food. We considered Daphnia as a useful experimental model for this investigation, since it is one of the most common model organisms in biological research (Ebert 2011); its physiology is well understood and it grows and reproduces rapidly. Furthermore, reproduction under favourable conditions is parthenogenetic, which allows us to exclude effects of genotypic variation on the response variable, isotopic variance (Lampert 2006). In contrast to Bearhop et al. (2004), we used the variance 
of stable carbon $\left(\delta^{13} \mathrm{C}\right)$ rather than of nitrogen $\left(\delta^{15} \mathrm{~N}\right)$ isotopes. This was done partly to reduce the effects of isotopic fractionation (McCutchan et al. 2003) on consumer isotope values, but also because $\delta^{13} \mathrm{C}$ is typically used to distinguish between different putative sources of energy fuelling consumers (France 1995).

As noted earlier, animals, in particular trophic generalists, do not necessarily feed on one resource type only throughout their life, but switch between several alternative resources. Within a population, switching to an alternative diet can occur synchronously for the whole population $\left(\mathrm{GA}_{\text {syn }}\right)$, e.g. with a habitat shift as it is commonly observed in many group-living animals ranging from wildebeest and zebra (Ben-Shahar and Coe 1992) to fish (e.g. ontogenetic niche shifts in perch, Hjelm et al. 2000). However, diet switches can also occur asynchronously within a population $\left(\mathrm{GA}_{\mathrm{as}}\right)$, if the population is spread over a heterogeneous habitat with patchy resource availability and different parts of the population utilize different food types at a given time (e.g. brown trout, Grey 2001). Information on such diet-switches of generalist populations can be obtained by examining several different tissues that reflect long and short-term foraging histories.

Following Bearhop et al. (2004), we hypothesized that $\mathrm{GB}$ and $\mathrm{GA}_{\text {as }}$ will have a large within-population isotopic variance as the short-turnover tissues are isotopically most influenced by the most recent feeding history, which, in turn, is different for individual members of an asynchronously diet-switching population (Fig. 3). Conversely, synchronously diet-switching populations would have small variances as all individuals feed on isotopically identical food sources (Fig. 3).
Hence, this study represents the first controlled empirical test of the applicability of the use of population-level measures of isotopic mean and variance for determining trophic niche width. This is achieved by 1) experimentally determining means and variances in consumer stable isotope values from specialist populations (SP, experiment 1), type A generalists populations $\left(\mathrm{GA}_{\text {long }}\right.$, long-term integrating tissue, experiment 1), short-term integrating tissue with synchronous $\left(\mathrm{GA}_{\mathrm{syn}}\right)$ and asynchronous $\left(\mathrm{GA}_{\mathrm{as}}\right)$ diet switching, and type $\mathrm{B}$ generalist populations (GB, experiment 2, Fig. 3), and 2) by deriving a model of the influence of dietary correlation time on variance of isotope values within natural populations. Together, these two approaches allow us to point to crucial prerequisites and potential caveats in the use of stable isotope variances to determine trophic niche width. We therefore conclude by presenting some general prescriptions that should be followed to avoid fallacies when applying the method of Bearhop et al. (2004), and our extensions of it to field data.

\section{Methods}

\section{Experimental approach}

By cultivating algae in the presence or absence of ${ }^{13} \mathrm{C}$ labelled bicarbonate, we were able to produce morphologically and qualitatively identical food items with widely differing isotopic values. Even though Daphnia are unselective filter-feeders with no individual food preferences (Lampert 2006), they can be used as model organisms to investigate the effects of dietary history on consumer stable
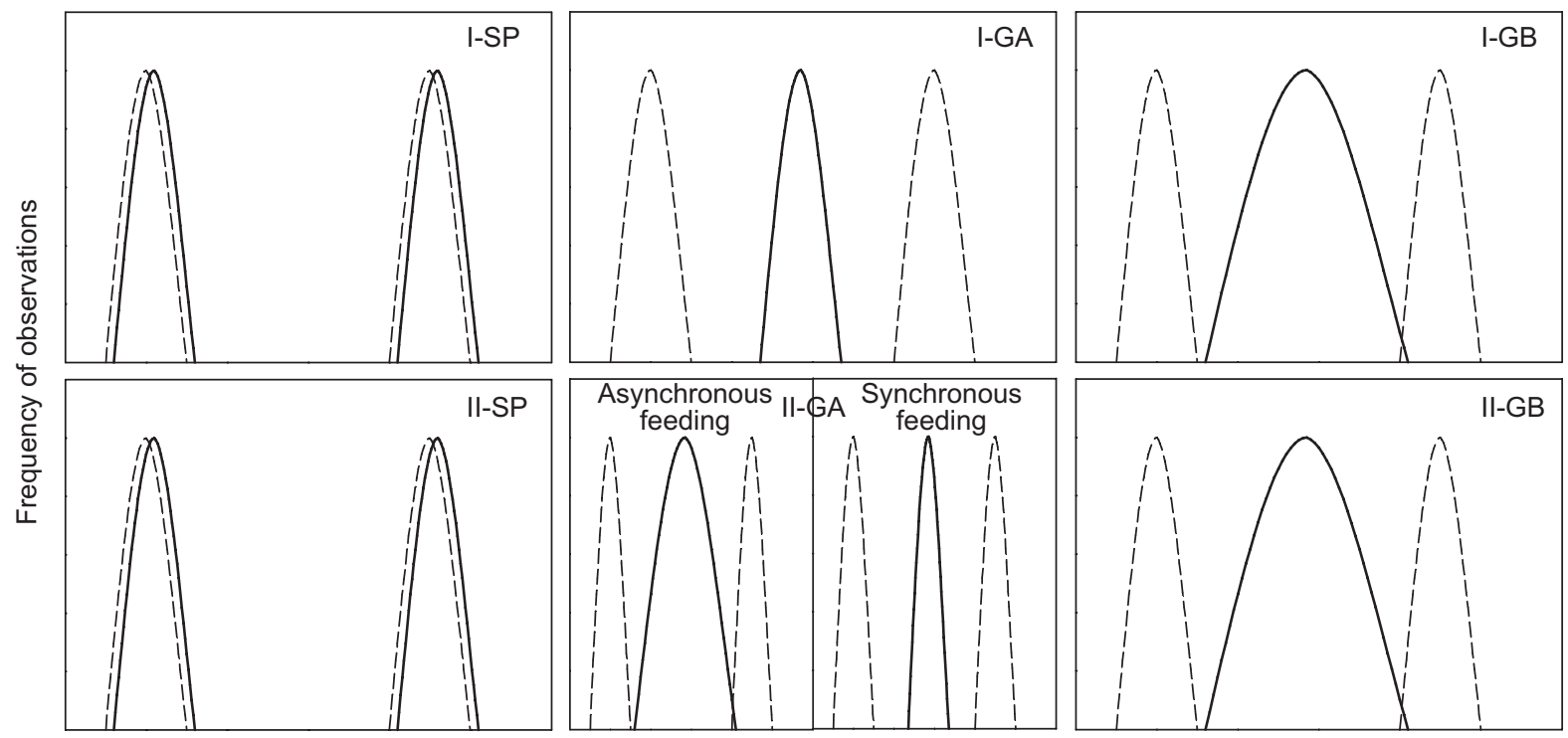

$\delta^{13} \mathrm{C}$

Figure 3. Expected isotopic frequency distributions for mean variances of tissue $\delta^{13} \mathrm{C}$ values for the three types of consumers, if two isotopically distinct food types are consumed: specialist population (SP), type A generalist population (GA), and type B generalist population (GB). Broken lines represent $\delta^{13} \mathrm{C}$ values from food items, solid lines represent consumer $\delta^{13} \mathrm{C}$ values. The upper panels (I) represent $\delta^{13} \mathrm{C}$ values in slow-turnover, long-time period integrating tissue; the lower panels (II) represent $\delta^{13} \mathrm{C}$ signatures in fast-turnover tissues. The split panel for GA shows the expected variances for synchronously and asynchronously feeding GA in short-term integrating tissue; the shift of the consumer's $\delta^{13} \mathrm{C}$ signatures to the right is due to trophic fractionation. 
isotope signatures by choosing the appropriate experimental design. By presenting different food sources either at the same or at different times, we were able to mimic the effects of different kinds of foraging behaviour and different relative time scales for diet switching and retention of material in consumer tissue. Long dietary correlation times, corresponding to short-term integrating tissue, were simulated by feeding Daphnia with food of known, fixed stable isotope values over a prolonged period (experiment 1). Short dietary correlation times, or long-term integrating tissues, were realised by switching the diet during the experiment (experiment 2). To mimic specialist consumers in our experiment, we fed groups of Daphnia with algae with divergent isotope values, allowing us to construct artificial populations of Daphnia with different feeding histories.

\section{Food culture and labelling}

The chlorophycean microalga Scenedesmus obliquus was used as sole food source for Daphnia pulicaria. Scenedesmus obliquus is commonly used as standard food for Daphnia sp. and allows daphnids to achieve high somatic growth rates (Lampert 2006). Two S. obliquus continuous cultures (chemostats) were set up for culturing with a dilution rate of $0.5 \mathrm{~d}^{-1}$ and with dilute (1:4) Z4 medium (Zehnder and Gorham 1960). The two chemostats were kept at constant irradiance $\left(100 \mu \mathrm{mol}\right.$ photons $\left.\mathrm{s}^{-1} \mathrm{~m}^{-2}\right)$ and temperature $\left(20^{\circ} \mathrm{C}\right)$. In one of the two chemostats, $0.5 \%$ of the added carbonate stock solution $\left(42.0 \mathrm{mg} \mathrm{l}{ }^{-1} \mathrm{NaHCO}_{3}\right)$ was replaced by $\mathrm{NaH}^{13} \mathrm{CO}_{3}$. This resulted in a shift in mean $( \pm \mathrm{SD}) \delta^{13} \mathrm{C}$ values of $S$. obliquus from $-15.4 \%$ o $( \pm 0.8)$ in the unlabelled culture to $8.5 \%$ o $( \pm 0.4)$ in the labelled culture in our first experiment, and from $-15.5 \%$ o $( \pm 0.3)$ in unlabelled S. obliquus to $4.1 \%$ o $( \pm 0.8)$ in the labelled culture in our second experiment. In order to assess whether labelling affected food quality of S. obliquus for the daphnids, the molar $\mathrm{C}: \mathrm{N}$ ratio and the fatty acid composition of labelled and unlabelled algae were analyzed, as described by Fink and Von Elert (2006a, 2006b).

\section{Pre-experimental Daphnia culture}

All experiments were conducted with four-day old juveniles of a clone of $D$. pulicaria, originally isolated from a pond near Konstanz, Germany, but kept in laboratory culture with $S$. obliquus as sole food for several years prior to the experiments (Alekseev and Lampert 2001). Juvenile D. pulicaria from synchronized third clutches were fed with unlabelled S. obliquus for three days after birth prior to each experiment.

\section{Experiment 1}

Determination of stable isotope means and variances for $S P$ and $G A_{\text {long }}$ populations (long-term integrating tissue)

The experiment was carried out in a temperature-controlled room at $20^{\circ} \mathrm{C}$ in glass beakers filled with 11 of $0.45-\mu \mathrm{m}$ filtered lake water (Schöhsee, northern Germany) and ran for three days. Daphnia were fed either 100\% unlabelled S. obliquus (in the following abbreviated as U), a 1:1 mixture of labelled and unlabelled S. obliquus (abbreviated here as M), or $100 \%$ labelled S. obliquus (abbreviated as L). In the context of dietary specialisation, animals feeding on $\mathrm{U}$ and $\mathrm{L}$ algae can be considered as two populations of specialist consumers, specialised on two different resources. The animals on the mixed $(\mathrm{M})$ algal diet can be considered as type A generalists (GA), as they indiscriminately feed on the mixture of $U$ and $L$ algae. Each treatment consisted of either 15 (L, M) or 21 (U) beakers (replicates) with 15 animals per beaker. The 15 Daphnia of each beaker were pooled for stable isotope analysis to reach the required weight for this analysis. Reported isotope values therefore reflect averages from 15 individuals. Food was supplied ad libitum $\left(1.5 \mathrm{mg} \mathrm{C}^{-1}\right)$ and animals were transferred daily into new food suspensions.

At the end of the experiment, D. pulicaria were transferred to filtered lake water for $4 \mathrm{~h}$ to allow gut clearance in order to avoid isotopic bias. Daphnia were then transferred into pre-weighed tin cups and dried for $24 \mathrm{~h}$ prior to determination of dry mass $( \pm 0.1 \mu \mathrm{g})$. Since juvenile Daphnia grow exponentially with respect to their body mass, and the juvenile growth rate is a good proxy of fitness (Lampert and Trubetskova 1996), we calculated somatic growth rates $(g)$ using the equation $g=\left(\ln \mathrm{W}_{\mathrm{t}}-\ln \mathrm{W}_{0}\right) / \mathrm{t}$, where $\mathrm{W}_{0}$ is the animals dry weight at the beginning and $\mathrm{W}_{t}$ the dry weight at the end of the experiment and $t$ the duration of the experiment in days. $\mathrm{W}_{0}$ was determined by separately weighing a subsample of the experimental animals (from the same clutch) at the beginning of the experiment. Carbon stable isotope ratios and C:N of both algae and Daphnia were determined by continuous flow isotope ratio mass spectrometry coupled to a mass spectrometer. Stable isotope ratios are given using the $\delta$ notation expressed in units per mil as follows: $\delta(\%)=$ $\left[\left(\mathrm{R}_{\text {sample }} / \mathrm{R}_{\text {standard }}\right)-1\right] \times 1000$, and $\mathrm{R}={ }^{13} \mathrm{C} /{ }^{12} \mathrm{C}$. The reference materials used was a secondary standard of known relation to the international standard of Vienna Pee Dee belemnite. Typical precision for a single analysis was $\pm 0.1 \%$. All data from the experiments were tested for homoscedasticity (Levene's test) and comparison between treatments done via one- or two-way analyses of variances (ANOVAs) using Statistica 6.0 (StatSoft Inc.). Results with a p $<0.05$ were considered significant.

\section{Experiment 2}

Determination of stable isotope means and variances for $G A_{s y n}$ (short-term integrating tissue; synchronous food source switching) and $G A_{\text {as }}$ (short-term integrating tissue; asynchronous food source switching)

In this experiment, Daphnia encountered three different treatments in which they received the three food sources with different isotopic values ( $\mathrm{U}, \mathrm{M}, \mathrm{L})$ but in different sequences (A, B, C; Table 1). Each treatment consisted of 20 replicates (beakers with 11 lake water) containing 15 Daphnia each. The experiment was run in a temperature controlled room at $20.7^{\circ} \mathrm{C}$ for 5 days. All analyses were performed as described above. One value was identified as an outlier due to an extreme $(>3)$ studentized residual value of -4.52 but was included in the data analysis as comparative analyses revealed that its inclusion had no discernible effect on our results. This experiment was designed to 
Table 1. Sequential feeding scheme for Daphnia in treatments A-C of experiment 2, following the suggestion of Bearhop et al. (2004). $\mathrm{U}=$ unlabelled food, $\mathrm{L}=$ labelled food, $\mathrm{M}=$ mixed food $(1: 1 \mathrm{U}: \mathrm{L})$.

\begin{tabular}{lccc}
\hline Day & Treatment A & Treatment B & Treatment C \\
\hline 1 & $\mathrm{M}$ & $\mathrm{M}$ & $\mathrm{L}$ \\
2 & $\mathrm{M}$ & $\mathrm{L}$ & $\mathrm{U}$ \\
3 & $\mathrm{~L}$ & $\mathrm{U}$ & $\mathrm{M}$ \\
4 & $\mathrm{U}$ & $\mathrm{M}$ & $\mathrm{M}$ \\
5 & $\mathrm{M}$ & $\mathrm{M}$ & $\mathrm{M}$ \\
\hline
\end{tabular}

create two different population types: $\mathrm{GA}_{\text {syn }}$ and $\mathrm{GA}_{\text {as }}$ populations. We obtained three different $\mathrm{GA}_{\text {syn }}$ populations by treating each treatment $(\mathrm{A}-\mathrm{C}$, all consisting of 20 beakers, respectively) as a single GA population that switched the diet synchronously. However, when we consider the individuals from all 60 beakers as a single population, three subpopulations (corresponding to treatments $\mathrm{A}-\mathrm{C}$ ) can be distinguished that switched their diets asynchronously $\left(\mathrm{GA}_{\mathrm{as}}\right)$.

\section{Model of consumer generalism and dietary correlation time}

In modelling changes in the $\delta^{13} \mathrm{C}$ value of Daphnia in response to changes in the $\delta^{13} \mathrm{C}$ value of its diet, we assume that the effect of consumed food on somatic $\delta^{13} \mathrm{C}$ decays exponentially over time. This assumption holds exactly if, for example, $\mathrm{C}$ atoms in the body tissue are replaced at random with $\mathrm{C}$ atoms of newly assimilated material, or when individuals grow exponentially in size, as is the case for juvenile Daphnia (Lampert and Trubetskova 1996), and body tissue is mostly added rather than replaced, as demonstrated for broad whitefish (Coregonus nasus) by Hesslein et al. (1993). Phenomenologically, we expect the assumption of exponential dilution of tissue to be valid over a wide range of situations. It leads to a simple model for Daphnia $\delta^{13} \mathrm{C}$ values of the form

$\delta^{13} \mathrm{C}_{\mathrm{daph}}(t)=r \int_{-\infty}^{t} e^{-r(t-\tau)} \delta^{13} \mathrm{C}_{\text {food }}(\tau) d \tau+\Delta_{C}$

where $r$ is the tissue dilution rate (i.e. the rate at which tissue and the associated isotopic values are diluted through addition and replacement), $\delta^{13} \mathrm{C}_{\text {daph }}(t)$ is the $\delta^{13} \mathrm{C}$ value of Daphnia at time $t, \delta^{13} \mathrm{C}_{\text {food }}(\tau)$ is the $\delta^{13} \mathrm{C}$ value of the food assimilated at time $\tau$ (assuming that the uptake rates are approximately constant over times of duration $1 / r$ ), and $\Delta_{C}$ represents the effect of fractionation (fractionation constant).

Our experimental protocol resolves time in units of days only. Model Eq. 1 is therefore appropriately re-written in the corresponding time-discrete form

$\delta^{13} \mathrm{C}_{\mathrm{daph}, \mathrm{t}}=\left(1-e^{-r}\right) \sum_{\tau=-\infty}^{t} e^{-r(t-\tau)} \delta^{13} \mathrm{C}_{\mathrm{food}, \tau}+\Delta_{C}$

where the replacement of the factor $r$ by $\left(1-e^{-r}\right)$ guarantees that, as expected, in the case of continuously feeding on a single resource the $\delta^{13} \mathrm{C}$ value is simply shifted by the fractionation constant.

Treatments $\mathrm{A}, \mathrm{B}$ and $\mathrm{C}$ of experiment 2 differ from treatment $M$ (mixed diet) of experiment 1 only in the two days where either unlabelled or labelled algae are fed to Daphnia (Table 1). Equation 2 therefore implies the following statistical models for the measured isotope values of treatments $\mathrm{M}, \mathrm{A}, \mathrm{B}$ and $\mathrm{C}$, which we fitted to the experimental results using the non-linear least-square fitting function nls of the software package R ( R Development Core Team):

$\delta^{13} \mathrm{C}=b+\epsilon$

for treatment $\mathrm{M}$, where $b$ represents the baseline result for feeding on mixed samples and $\epsilon$ a random residual, and

$\delta^{13} \mathrm{C}=b+a\left(1-e^{-r}\right)\left(-0.5 e^{-r d}+0.5 e^{-r(d+1)}\right)+\epsilon$

for treatments A, B, C (Table 1). The experimental parameter $d$ represents the delay between the day of feeding on unlabelled algae and of sampling, which is varied in the three treatments. The parameter $a$, scaling the effect strength, would ideally equal the difference in isotope values between labelled and unlabelled algae (17.6), however, to absorb uncertainties in the effective time of sampling (i.e. the effective delay $d$ ), we treat $a$ as a free model parameter, to be fitted to the data, just as baseline value $b$ and dilution rate $r$.

\section{Results}

\section{Labelling and food quality}

The ${ }^{13} \mathrm{C}$-labelling resulted in slightly altered molar C:N ratios $\left(\mathrm{F}_{2,34}=108.6, \mathrm{p}<0.001\right)$ in algae (mean $\pm \mathrm{SD} 4.7 \pm 0.1$ for labelled algae, $5.0 \pm 0.1$ for unlabelled Scenedesmus obliquus). However, as it is to be expected for a stoichiometrically homeostatic consumer (Persson et al. 2010), the daphnids' molar C:N ratios were not significantly affected by the different food sources (mean \pm SD $4.5 \pm 0.1$ for L, $\mathrm{M}$ and $\mathrm{U} ; \mathrm{F}_{2,48}=0.16, \mathrm{p}=0.86$ ) and the $\mathrm{C}: \mathrm{N}$ variance of the animals was stable between food treatments (Levene's test: $\left.\mathrm{F}_{2,46}=0.827, \mathrm{p}=0.44\right)$. The fatty acid composition (\% of total fatty acids) of S. obliquus was also not affected by labelling (two-way ANOVA: 'label' $\mathrm{F}_{1,24}=0.0, \mathrm{p}=1.0$, 'fatty acid' $\mathrm{F}_{5,24}=3577.5, \mathrm{p}<0.001$ ). Furthermore, there were no significant differences in $D$. pulicaria growth rates between feeding regimes in the first experiment (one-way ANOVA, $\left.\mathrm{F}_{2,48}=2.7, \mathrm{p}=0.08\right)$. Mean $( \pm \mathrm{SD})$ juvenile growth rate was $0.40 \pm 0.02 \mathrm{~d}^{-1}$ in the daphnids fed unlabelled algae, $0.41 \pm 0.02 \mathrm{~d}^{-1}$ with labelled algae, and $0.40 \pm 0.02 \mathrm{~d}^{-1}$ in those fed the mixture of labelled and unlabelled $S$. obliquus. In experiment 2, the somatic growth rates in treatment $\mathrm{A}\left(0.51 \pm 0.02 \mathrm{~d}^{-1}\right)$ were slightly, but significantly (one-way ANOVA: $\mathrm{F}_{2,57}=6.8, \quad \mathrm{p}<0.01$ ) higher than in treatments $B$ and $C$ (both $0.50 \pm 0.02 \mathrm{~d}^{-1}$ ). This was probably due to slight imbalance in the temperature distribution within the experimental chamber, since treatments $\mathrm{B}$ and $\mathrm{C}$ were situated closer to the cooling system, resulting in slightly $\left(0.11^{\circ} \mathrm{C}\right)$ reduced water temperatures. Nevertheless, the differences were very small and we therefore feel that there is little evidence for differences in food quality between the treatments. 


\section{Experiment 1}

Experimentally determining stable isotope means and variances for SP and $G A_{\text {long }}$ (long-term integrating tissue) Experiment 1 resulted in two 'specialist' populations (sensu Bearhop et al. 2004): one fed unlabelled (U), one labelled (L) algae. The isotopic difference in resource resulted in a marked difference in the consumers' carbon isotope values: At the beginning of the experiment, all Daphnia had the same mean $( \pm S D ; n=4) \delta^{13} \mathrm{C}$ of $-17.4 \%$ o $( \pm 0.1)$. After three days fed with either $U\left(\delta^{13} \mathrm{C}-15.4 \% \pm \pm 0.8\right)$ or L $\left(\delta^{13} \mathrm{C} 8.5 \% \pm 0.4\right)$ S. obliquus, Daphnia's mean $\delta^{13} \mathrm{C}$ $( \pm S D)$ was $-16.3 \%$ o $( \pm 0.16 ; n=21)$ in $U$ and $1.1 \%$ $( \pm 0.27 ; \mathrm{n}=15)$ in the $\mathrm{L}$ treatment. Mean $\delta^{13} \mathrm{C}$ differed between treatments (Fig. 4: $\mathrm{F}_{1,34}=59972, \mathrm{p}<0.001$ ). In addition to the two specialist populations, this experiment also yielded one GA population fed a 1:1 mixture of labelled and unlabelled food (treatment $M)$. The mean $\delta^{13} \mathrm{C}( \pm \mathrm{SD}$; $\mathrm{n}=13)$ of the mixed algal resource was $-3.4 \%$ o ( \pm 0.54$)$. The mean $\delta^{13} \mathrm{C}( \pm \mathrm{SD} ; \mathrm{n}=15)$ of the Daphnia fed with this mixture for three days was $-7.3 \%$ o $( \pm 0.24)$. Mean $\delta^{13} \mathrm{C}$ differed between all three populations (one-way ANOVA: $\left.\mathrm{F}_{2,48}=27901, \mathrm{p}<0.0001\right)$, but $\delta^{13} \mathrm{C}$ variance was always small and did not differ between populations (Levene's test: $\mathrm{F}_{2,48}=2.38, \mathrm{p}=0.104$; Fig. 4).

\section{Experiment 2}

\section{Experimentally determining stable isotope means and variances for $\mathbf{G A}_{\text {short }}$ (short-term integrating tissue)}

Daphnia were subject to three dietary treatments by feeding them either unlabelled (U) or labelled (L) S. obliquus, or a $1: 1$ mixture of $U$ and $L$ algae having an intermediate $\delta^{13} \mathrm{C}$ (M). Mean algal $\delta^{13} \mathrm{C}( \pm \mathrm{SD})$ was $-15.5 \% \pm 0.3$ for $\mathrm{U}(\mathrm{n}=25), 4.1 \% 0 \pm 0.8$ for $\mathrm{L}(\mathrm{n}=25)$ and $-5.5 \% \mathrm{t}$ 0.4 for $M(n=24)$. Mean $( \pm S D) \delta^{13} \mathrm{C}$ of Daphnia was $-18.1 \%$ o $( \pm 0.5, \mathrm{n}=2)$ at the beginning of the experiment.

After five days mean ( \pm SD) Daphnia $\delta^{13} \mathrm{C}$ was $-9.8 \%$ $( \pm 0.2, \mathrm{n}=20)$ in treatment $\mathrm{A},-8.7 \%$ o $( \pm 0.4, \mathrm{n}=20)$ in treatment $\mathrm{B}$ and $-8.2 \%$ o $( \pm 0.2, \mathrm{n}=20)$ in treatment $\mathrm{C}$, i.e. the variance (squared SD) was relatively small within each GA population, if food switching occurred synchronously as compared to a $\mathrm{GA}_{\text {as }}$ population (Fig. 4). If these three treatments were considered as three generalist subpopulations that fed asynchronously on different resources, then the calculated mean $( \pm S D)$ was $-8.9 \%$ o $( \pm 0.7, n=60$, Fig. 4). This revealed that variance within $\mathrm{GA}_{\mathrm{as}}$ populations was large when a rapid turnover tissue and asynchronous feeding was considered.

Comparison of the isotopic variance in this $\mathrm{GA}_{\text {short }}$ population (experiment 2) with the $\mathrm{GA}_{\text {long }}$ population where we consider a sampling of long-term integrating tissue (experiment 1 ), showed greater variance in experiment 2 (Levene's test: $\mathrm{F}_{1,73}=30.6, \mathrm{p}<0.0001$ ), following the predictions of Bearhop et al. (2004, Fig. 3, 4).

\section{Fitting of the model for tissue dilution}

Model parameters in Eq. (3a, b), estimated from the experimental results, their standard errors, and confidence intervals are listed in Table 2 . The residual standard deviation, quantifying the empirical accuracy, was 0.27 . Residuals were
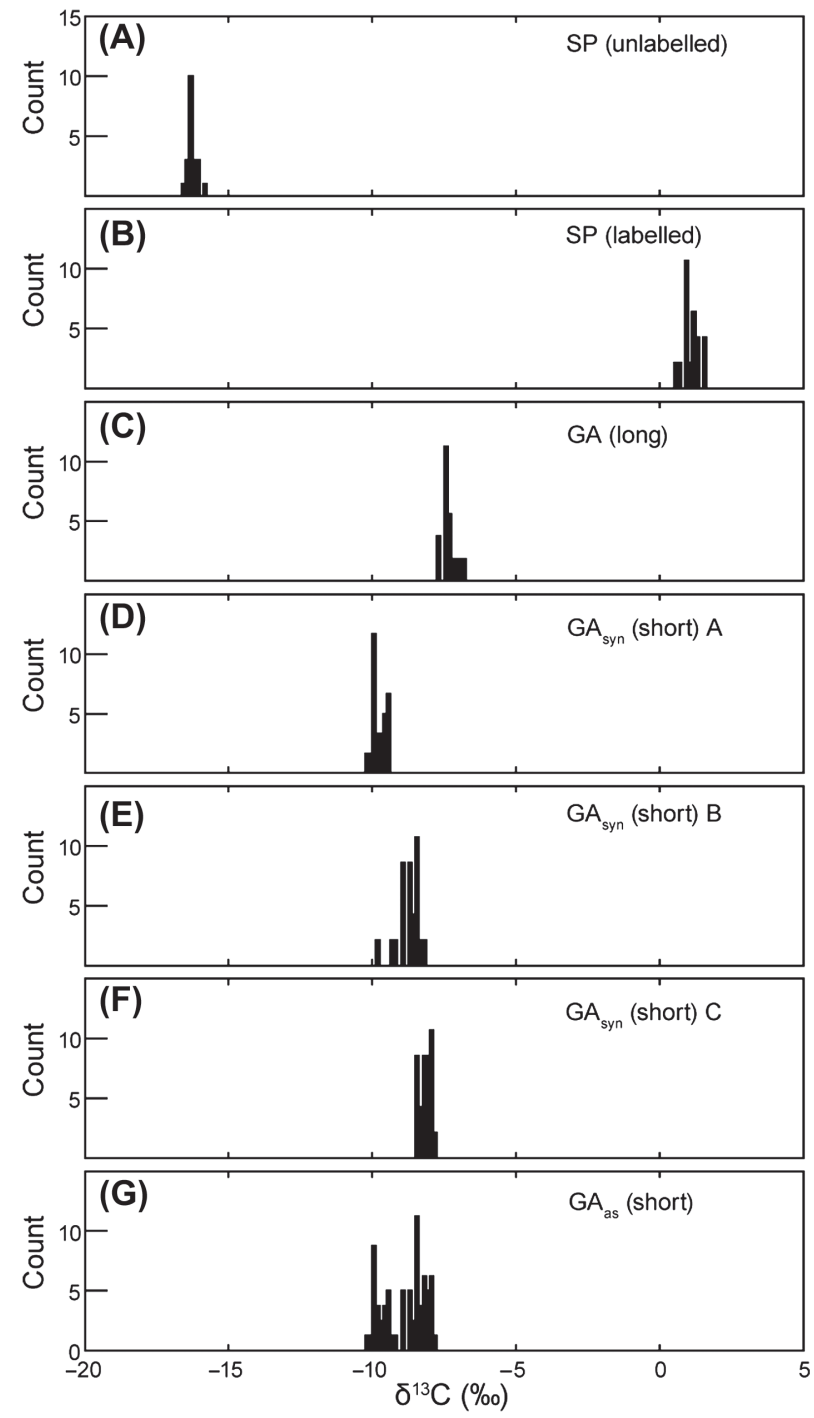

Figure 4. Frequency histograms of $\delta^{13} \mathrm{C}$ values (from the experiments) of two specialist populations (SP, fed unlabelled and labelled food, respectively), long-term integrating tissue of one type A generalist population $\left(\mathrm{GA}_{\text {long }}\right)$, short-term integrating tissue of three GA populations feeding synchronously within the populations $\left(\mathrm{GA}_{\text {syn }}, \mathrm{A}-\mathrm{C}\right)$, and a short-term integrating tissue of one GA population feeding asynchronously $\left(\mathrm{GA}_{\mathrm{as}}\right)$. Data for $\mathrm{SP}_{\text {unlabelled }}$ $(\mathrm{n}=21), \mathrm{SP}_{\text {labelled }}(\mathrm{n}=15)$ and $\mathrm{GA}_{\text {long }}(\mathrm{n}=15)$, were taken from the experiment 1; GA $A_{\text {short }}$ A $(n=20), \mathrm{GA}_{\text {short }} \mathrm{B}(\mathrm{n}=19), \mathrm{GA}_{\text {short }} \mathrm{C}$ $(\mathrm{n}=20)$ and $\mathrm{GA}_{\text {asyn }}(\mathrm{n}=59)$ were taken from experiment 2 .

normally distributed according to the Lilliefors (adjusted Kolmogorov-Smirnov) test $(\mathrm{D}=0.06, \mathrm{p}=0.747)$. As noted above, a Q-Q-plot of residuals indicates that one sample in treatment B of experiment 2 was an outlier. However, since removing this data point shifts the confidence intervals only by small amounts, and its classification is not entirely clear, the data point was retained.

Table 2. Maximum likelihood fit of the model given by Eq. 3a-b to laboratory results.

\begin{tabular}{lccccc}
\hline Variable & Unit & Best fit & Std. error & $2.5 \%$ & $97.5 \%$ \\
\hline $\mathrm{a}$ & $\%$ o & 82.7 & 4.2 & 75.0 & 92.2 \\
$\mathrm{~b}$ & $\%$ o & -7.33 & 0.07 & -7.47 & -7.19 \\
$\mathrm{r}$ & day $^{-1}$ & 0.549 & 0.041 & 0.472 & 0.638 \\
\hline
\end{tabular}




\section{Discussion}

Following earlier observations by e.g. Fry et al. (1978), Bolnick et al. (2003), Matthews and Mazumder (2004) and others, Bearhop et al. (2004) developed a hypothesis that the variance in stable isotope ratios of consumer tissues can be used to determine the trophic niche width of consumer populations. For this approach to be valid, the authors stated the following conditions: 1) the possible food sources must be isotopically distinct; 2) each food source must be relatively invariable over time; and 3) the tissue analysed must reflect the period over which the niche width is expressed. If these assumptions are fulfilled, Bearhop et al. (2004) predicted that it is possible to distinguish between specialist populations (SP), and two types of generalist populations (GA and GB, Fig. 1,3) by analysing the variance of stable isotope ratios of appropriate consumer's tissues.

More formally, the distinction between generalists and specialists can be regarded as two extremes in a range of dietary correlation times. In these extreme cases, the generalist switches diets continuously while the specialist never switches. Field ecologists require information that allows them to assess the relative importance of assimilated prey on the isotopic variance recorded from wild populations. Here we have determined the factors driving dietary correlation time, which in turn influences population-wide isotopic variance. To facilitate this, we here created a model of isotopic variance dependent on dietary correlation time within a (model) population. The model makes the assumption that the impact of food on consumer isotope ratios decays approximately exponentially with the time since assimilation. We find our experimental data consistent with this model and that the decay rate can be measured to good precision.

As predicted, our results from Experiment 1 clearly demonstrated specialist populations (Fig. 4A, B) had reduced isotopic variance compared to generalist populations (Fig. 4C, G). However, one should note that the variances were measured under laboratory conditions that largely eliminated environmental and genetic variability. Variances were further reduced by pooling 15 individuals in each sample. In the field, all variance will be enhanced due to environmental and genetic variability among individuals. In principle, this should act as a constant offset, keeping differences between variances unaffected.

During our experiments, the Daphnia populations were not in equilibrium with the food, as our experimental period was too short to permit full tissue turnover (Grey 2000). Thus, our experiment imitated a natural situation, e.g. following a marked isotopic shift. Such a shift would occur for example during lake turnover, when lake stratification breaks down in the autumn/winter, and previously distinct water masses are mixed. This makes new food materials available to zooplankton (Zohary et al. 1994, Harrod and Grey 2006, Perga and Gerdeaux 2006). Variance in stable isotope values was also small in type A generalist populations $\left(\mathrm{GA}_{\text {long }}\right)$, when we imitated sampling of long-term integrating tissue. We imitated this type A generalist population by feeding the Daphnia with two isotopically different food sources. As Daphnia are non-selective feeders and as the isotopically different food particles did not differ in any feature, every beaker represented a generalist individual within a population (treatment). Our analysis shows that the variances are equal in the two $\mathrm{SP}$ and GA, again in accordance with the prediction of Bearhop et al. (2004). Thus, taking only the variances of the stable isotope ratios into account, it would not have been possible to differentiate between these two types of populations. However, by taking also the mean stable isotope ratio into account and by knowing the stable isotope values of the potential food sources, we can reliably distinguish between the two population types. This is due to the fact that the mean stable isotope ratio of consumers corresponds well to the mean stable isotope value of the food source. If more than one food source is ingested, then the mean also depends on the proportion of the ingested food sources.

Many animal species, both trophic generalists but also species with pronounced ontogenetic diet shifts, do not feed on only one resource type throughout their life, but switch between several alternative resources. Within a population, switching to an alternative diet can occur synchronously for the whole population, e.g. with a habitat shift (Ben-Shahar and Coe 1992, Hjelm et al. 2000); or asynchronously within a population, if different parts of the population utilise different food patches at a given time (Harrod et al. 2010). Information on such diet-switches of generalist populations can be obtained by examining several different tissues that reflect long- and short-term foraging histories. We studied this in our second experiment in which we simulated two different GA population types: If each beaker within a treatment $(\mathrm{A}, \mathrm{B}$ or $\mathrm{C})$ was taken as a $\mathrm{GA}$ population we simulate three populations $(\mathrm{A}-\mathrm{C})$ that underwent a synchronous diet switch. In contrast, all beakers together can be interpreted as belonging to a single population consisting of three generalist subpopulations (the three treatments) that switched their food source asynchronously. While our synchronously feeding GA population $\left(\mathrm{GA}_{\text {syn }}\right)$ had a small variance, the $\mathrm{GA}_{\text {as }}$ population feeding asynchronously yielded a large variance (Fig. 4), permitting the two different population types to be differentiated.

Combining the results from the two experiments, we suggest that one can not only reliably differentiate between SP and GA populations, but also between asynchronous and synchronous diet-switching modes in GA populations. However, this is limited to situations where short-term integrating tissues are available (Fig. 3).

\section{Modelling the $\delta^{13} \mathrm{C}$ response function}

The central result of fitting the response function (Eq. 1) to the experimental data is an estimate of the tissue dilution rate (with standard error in parentheses) $r=0.549 \mathrm{~d}^{-1}$ (0.041) which is, next to the constant fractionation offset, the only parameter entering this generic model. We find the dilution rate to be equal or slightly larger than the growth rate $g=0.4-0.5 \mathrm{~d}^{-1}$. A small difference of the order of $0.1 \mathrm{~d}^{-1}$ can indeed be expected in view of the tissue turnover rates of $0.1 \mathrm{~d}^{-1}$ found by Grey (2000) for adult Daphnia which show only limited growth.

From Eq. 1, it is immediately clear that Daphnia individuals that feed on all potential food items within time intervals much shorter than the dilution time of $r^{-1} \approx 2$ days 
have a $\delta^{13} \mathrm{C}$ value that simply represents the isotopic average of their diets, offset by trophic fractionation. In the opposite case, when individuals continuously feed on a single food type over time intervals that are long compared to $r^{-1}$, their $\delta^{13} \mathrm{C}$ value corresponds with the food type they are currently feeding on, again offset by trophic fractionation. However, Eq. 1 also makes quantitative predictions for intermediate cases. Assume, for example, that the $\delta^{13} \mathrm{C}$ values of the food items of Daphnia, weighted by diet proportions, are approximately normally distributed with variance $V_{\text {food }}$, and that, as an individual browses different food categories, the momentary $\delta^{13} \mathrm{C}$ value of its diet can be modelled by a mean-reverting random walk (Ornstein-Uhlenbeck process) with reversion rate $k$. The correlation time of this process, 1/k (Gardiner 1983), gives the dietary correlation time. Equation 1 effectively describes a linear filter, smoothing the time series of $\delta^{13} \mathrm{C}_{\text {food }}(t)$ with a filter kernel $r e^{-r t}$, which has a power gain (the squared modulus of its Fourier transform) that depends on angular frequency $\omega$ as $r^{2} /\left(r^{2}+\omega^{2}\right)$. The variance $V_{\text {cons }}$ of the $\delta^{13} \mathrm{C}$ value of individuals can therefore be computed as the integral, over all radial frequencies $\omega$, of the product of the power spectrum of the Ornstein-Uhlenbeck process (given by $\pi^{-1} k V_{\text {food }}$ l $\left.\left(k^{2}+\omega^{2}\right)\right)$ and the power gain of the filter kernel. This yields

$$
V_{\text {cons }}=\int_{-\infty}^{\infty} \frac{1}{\pi} \frac{k V_{\text {food }}}{k^{2}+\omega^{2}} \frac{r^{2}}{r^{2}+\omega^{2}} d \omega=\frac{V_{\text {food }}}{1+\frac{k}{r}}
$$

Figure 5 illustrates the dependence of $V_{\text {cons }}$ on $k / r$ predicted by this model for a hypothetical field study. Equation 4 contains the previously discussed limiting cases: when food sources are varied quickly $(k>>r)$ all individuals have similar $\delta^{13} \mathrm{C}$ values and $V_{\text {cons }}$ is much smaller than $V_{\text {food }}\left(V_{\text {cons }} \approx\right.$ $\left.r V_{\text {food }} / \mathrm{k}<<V_{\text {food }}\right)$, while in the opposite case $(k<<r)$ the two variances are practically identical.

Together with an example for the expected dependence of $V_{\text {food }}$ on dietary correlation time, Fig. 5 also shows estimates of expected measurement errors and biases. The

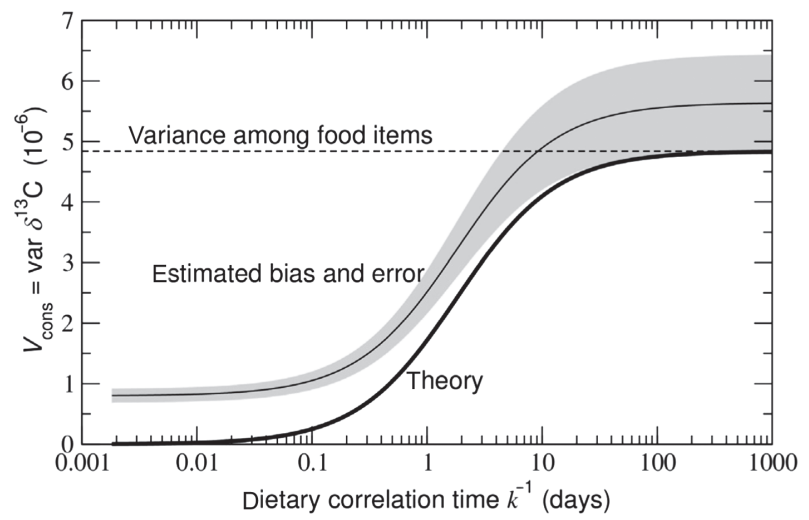

Figure 5. Predicted variance of $\delta^{13} \mathrm{C}$ values among Daphnia individuals as a function of the dietary correlation time (thick solid line). To illustrate typical experimental uncertainties, the thin solid line adds variance due to non-trophic variability, and the shaded area represents the expected statistical errors (1 SE) in the case that 100 individuals are separately analysed. The dashed line gives the variance corresponding to an assumed standard deviation in $\delta^{13} \mathrm{C}$ among diet items of $2.2 \%$ (Vuorio et al. 2006). curve is computed from Eq. 4 with $r$ set to the value and $V_{\text {food }}=2.2 \%$, which corresponds to the variance of $\delta^{13} \mathrm{C}$ values among phytoplankton taxa reported by Vuorio et al. (2006) for a freshwater lake. As a simple estimate of additional contributions to $V_{\text {cons }}$ due to measurement uncertainty and non-trophic variation among individuals, we used the mean over the cases $\mathrm{U}, \mathrm{L}$, and $\mathrm{M}$ in Experiment 1 of the products of observed sample variances (Table 3) and the numbers of Daphnia per sample, giving $0.8 \times 10^{-6}$. Expected standard errors of empirically determined variances were then computed for a hypothetical sample size of $n=100$, using the formula $2^{1 / 2}(\mathrm{n}-1)^{-1 / 2} V_{\text {cons }}$, which is the exact result for the standard error of a variance, estimated using the standard formula from normally distributed sample values.

Araújo et al. (2007) performed a related analysis, in which the degree of individual specialisation of frogs in a field study was estimated from isotopic variance among individuals and compared against the degree of individual specialisation that would have been deduced from the momentary stomach content of individuals sampled in the field. Depending on the species studied, agreements between the two estimates were found, but also deviations in either direction. The approach of Araújo et al. differs from ours in that their model for isotopic variance implicitly assumes diets to be fixed over times of the order of magnitude of the tissue integration time (type GB or $\mathrm{GA}_{\text {short }}$ ), but instead contains a parameter controlling the degree of individual variation in the composition of these diets. The difference between the two models corresponds to different models of foraging behaviour. While our approach assumes that a consumer's next prey choice is biased to be similar to recent prey choices (and DCT parameterises this bias), the model of Araújo et al. (2007) implies that variation in individual prey preference are in effect statistically independent of the temporal order of previous intakes. Instead, they could be controlled by phenotypic variation among individuals (Fermon and Cibert 1998, Robinson et al. 1993, Svanbäck and Bolnick 2005). Both behavioural models are plausible as they stand. Only detailed studies of foraging behaviour can reveal the relative degree to which they are realised in nature, and hence which analytic approach of isotopic variation is more appropriate.

Table 3. Isotopic $\left(\delta^{13} \mathrm{C}\right)$ values of Daphnia pulicaria (experiment 1$)$ at the start and the end of the experiment. Throughout the experiment, Daphnia were fed either unlabelled Scenedesmus obliquus $(\mathrm{U}),{ }^{13} \mathrm{C}$-labelled $S$. obliquus (L) or a 1:1 mixture of $\mathrm{U}$ and $\mathrm{L}(\mathrm{M})$; The last column gives the expected $\delta^{13} \mathrm{C}$ signature of Daphnia when in equilibrium with their new food; Note that animals fed $U$ and $L$ represent specialist populations (SP), while animals fed $M$ represent a type $A$ generalist population (long-term tissue turnover, GA long$_{\text {) }}$.

\begin{tabular}{|c|c|c|c|c|}
\hline \multirow[b]{2}{*}{ Treatment } & \multirow[b]{2}{*}{ Food label } & \multicolumn{2}{|c|}{$\begin{array}{c}\text { Non-equilibrium } \\
\text { Daphnia } \delta^{13} \mathrm{C} \\
\text { (measured) }\end{array}$} & \multirow{2}{*}{ 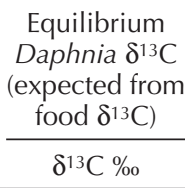 } \\
\hline & & $\delta^{13} \mathrm{C} \%$ & variance & \\
\hline & start & -17.4 & 0.080 & \\
\hline SP & unlabelled (U) & -16.3 & 0.025 & -15.4 \\
\hline SP & labelled (L) & 1.1 & 0.070 & 8.5 \\
\hline $\mathrm{GA}_{\text {long }}$ & mixed $(M)$ & -7.3 & 0.057 & -3.4 \\
\hline
\end{tabular}




\section{Consequences for the determination of trophic niche width}

The stable isotope approach for determining trophic niche width proposed by Bearhop et al. (2004) is a very appealing method, as it is less labour intensive than conventional gut content analysis and depending on the tissues sampled can reflect longer periods of consumer feeding behaviour. It has been used in a series of recent field studies which confirmed its successful applicability (Jaeger et al. 2010, Willson et al. 2010). Additionally, by choosing the appropriate tissue, this method delivers a good time-integrated measurement of niche width, which is difficult to achieve with the conventional methods. This is of particular importance when seasonally synchronized diet shifts of specialist populations occur which can only be interpreted when analyzing tissues with different integration times (Martinez del Rio et al. 2009). When Syväranta and Jones (2008) investigated their field data from fish with respect to the predictions of Bearhop et al. (2004), they found that the variances in stable isotope ratios in fish changed after mass removal of fish from an originally overpopulated lake and they interpreted the results as a broadening of the niche due to competitive release. However, none of these previous studies gave any direct evidence that the niches actually widened but only made indirect conclusions based on the prediction of Bearhop et al. (2004). While these previous studies are important starting points to indirectly assess the validity of the model in natural systems, our work aims to present more direct evidence from a controlled laboratory system. Our data clearly demonstrate that variation in isotopic variance between different population types is closely associated with the trophic history of the populations.

In closing, we propose a standard workflow for the use of dietary correlation time as a useful tool to quantify the position of a population on the 'specialist-/generalist-individuals' axis. We point out important issues and potential caveats that isotope ecologists should consider:

1. Determine the diet of the target population of consumers. To avoid circularity of the analysis, this will generally require the use of traditional methods (e.g. observations of foraging, analyses of gut/stomach content, faeces).

2. Determine the population-level isotopic variance of the diet $V_{\text {food }}$, weighted by relative contributions.

3. Determine the tissue dilution rate $r$ in laboratory studies or from the literature, or, for juveniles, estimate it from the somatic growth rate $g$ as $r \approx g$, either for the targeted organisms or for similar species.

4. Determine the population-level isotopic variance among individuals $V_{\text {cons }}$ of the target population in the field.

5. Estimate the dietary reversion rate $k$ by solving Eq. 4, i.e. $k=r\left[\left(V_{\text {food }} / V_{\text {cons }}\right)-1\right]$. The dietary correlation time equals $k^{-1}$.

6. Understand potential errors. The dependence of dietary correlation time on $r$ is rather robust: according to the standard propagation-of-errors law, the relative error in dietary correlation time contributed by errors in $r$ is of the same magnitude as the relative error in $r$ itself. The dependence of $k$ on $V_{\text {food }}$ and $V_{\text {cons }}$ is more sensitive. For pronounced individual generalism, that is,
$V_{\text {food }} \approx V_{\text {cons }}$ and $V_{\text {food }} / V_{\text {cons }} \approx 1$, only the semi-quantitative conclusion that $k<<r$ will be possible, because $k$ is then given by the difference between two similar values. Use of tissues with shorter integration time might mitigate this constraint. As illustrated in Fig. 5, statistical errors in $V_{\text {cons }}$ can be substantial, even with a sample size of 100 individuals. Besides, $V_{\text {cons }}$ will generally contain an unintended additive contribution of non-dietary origin (e.g. genetic variation). This contribution can be estimated in laboratory experiments, hence allowing determination of bounds on the resulting biases on dietary reversion rate $k$ and dietary correlation time $k^{-1}$.

In summary, our results support Bearhop et al.'s (2004) proposed use of stable isotope ratios to distinguish between populations that differ in their trophic niche. However, we urge that users also take mean stable isotope ratio values into account, as this will permit differentiation between SP and GA populations, when only short-term integrating tissues are available. This is particularly important in the case of small-bodied animals that are short lived, where only whole-body samples can be obtained. The stable isotope method provides a valuable means to distinguish between different consumption patterns ranging from true specialists through to true generalists. Nevertheless, it should be considered that it gives no information about the actual food items consumed, which can be investigated using the traditional approach of gut content analyses, or estimated from isotope mixing models, where the isotopic values of different prey resources are known (Phillips and Gregg 2001). Further studies are required to test the reliability of the method in a range of ecological settings, combining niche width estimates derived from stable isotope values and conventional gut content analysis.

Acknowledgements - This work would not have been possible without the support and funding by Winfried Lampert and the Max Planck Society. We would like to thank Heinke Buhtz and Elke Blohm-Sievers for excellent technical assistance. The contribution by AGR was carried out under a Beaufort Marine Research Award under the Sea Change Strategy and the Strategy for Science Technology and Innovation (2006-2013), with the support of the Marine Institute, funded under the Marine Research Sub-Programme of the Irish National Development Plan 2007-2013.

\section{References}

Alekseev, V. and Lampert, W. 2001. Maternal control of resting-egg production in Daphnia. - Nature 414: 899-901.

Araújo, M. et al. 2007. Using d13C stable isotopes to quantify individual-level diet variation. - Oecologia 152: 643-654.

Bearhop, S. et al. 2004. Determining trophic niche width: a novel approach using stable isotope analysis. - J. Anim. Ecol. 73: 1007-1012.

Ben-Shahar, R. and Coe, M. J. 1992. The relationships between soil factors, grass nutrients and the foraging behavior of wildebeest and zebra. - Oecologia 90: 422-428.

Bolnick, D. I. et al. 2003. The ecology of individuals: incidence and implications of individual specialization. - Am. Nat. 161: $1-28$. 
Cabana, G. and Rasmussen, J. B. 1994. Modeling food-chain structure and contaminant bioaccumulation using stable nitrogen isotopes. - Nature 372: 255-257.

Ebert, D. 2011. A genome for the environment. - Science 331: $539-540$

Fermon, Y. and Cibert, C. 1998. Ecomorphological individual variation in a population of Haplochromis nyererei from the Tanzanian part of Lake Victoria. - J. Fish Biol. 53: 66-83.

Fink, P. and Von Elert, E. 2006a. Food quality of algae and cyanobacteria for the freshwater gastropod Bithynia tentaculata: the role of polyunsaturated fatty acids. - Verh. Int. Verein. Limnol. 29: 1235-1240.

Fink, P. and Von Elert, E. 2006b. Physiological responses to stoichiometric constraints: nutrient limitation and compensatory feeding in a freshwater snail. - Oikos 115: 484-494.

France, R. 1995. Carbon-13 enrichment in benthic compared to planktonic algae: foodweb implications. - Mar. Ecol. Prog. Ser. 124: 307-312.

Fry, B. et al. 1978. Grasshopper food web analysis: use of carbon isotope ratios to examine feeding relationships among terrestrial herbivores. - Ecology 59: 498-506.

Gardiner, C. W. 1983. Handbook of stochastic methods. - Springer.

Grey, J. 2000. Trophic fractionation and the effects of diet switch on the carbon stable isotope signature of pelagic consumers. - Verh. Int. Ver. Limnol. 27: 3187-3191.

Grey, J. 2001. Ontogeny and dietary specialization in brown trout (Salmo trutta L.) from Loch Ness, Scotland, examined using stable isotopes of carbon and nitrogen. - Ecol. Freshwater Fish 10: $168-176$.

Harrod, C. and Grey, J. 2006. Isotopic variation complicates analysis of trophic relations within the fish community of Plußsee: a small, deep, stratifying lake. - Arch. Hydrobiol. 167: 281-299.

Harrod, C. et al. 2010. Phenotype-environment correlations in a putative whitefish adaptive radiation. - J. Anim. Ecol. 79: 1057-1068.

Hesslein, R. H. et al. 1993. Replacement of sulfur, carbon, and nitrogen in tissue of growing broad whitefish (Coregonus nasus) in response to a change in diet traced by delta-S-34, delta-C-13 and delta-N-15. - Can. J. Fish. Aquat. Sci. 50: 2071-2076.

Hjelm, J. et al. 2000. Growth, morphological variation and ontogenetic niche shifts in perch (Perca fluviatilis) in relation to resource availability. - Oecologia 122: 190-199.

Hobson, K. A. 1999. Tracing origins and migration of wildlife using stable isotopes: a review. - Oecologia 120: 314-326.

Hobson, K. A. and Clark, R. G. 1992. Assessing avian diets using stable isotopes. I. Turnover of ${ }^{13} \mathrm{C}$ in tissues. - Condor 94: $181-188$.

Hyslop, E. J. 1980. Stomach contents analysis - a review of methods and their application. - J. Fish Biol. 17: 411-429.

Jaeger, A. et al. 2010. Use of stable isotopes to quantify seasonal changes of trophic niche and levels of population and individual specialisation in seabirds. - Mar. Ecol. Prog. Ser. 401: 269-277.

Lampert, W. 2006. Daphnia: model herbivore, predator and prey. - Pol. J. Ecol. 54: 607-620.
Lampert, W. and Trubetskova, I. 1996. Juvenile growth rate as a measure of fitness in Daphnia. - Funct. Ecol. 10: 631-635.

Martinez del Rio, C. et al. 2009. Dietary and isotopic specialization: the isotopic niche of three Cinclodes ovenbirds. - Oecologia 161: 149-159.

Matthews, B. and Mazumder, A. 2004. A critical evaluation of intrapopulation variation of delta C-13 and isotopic evidence of individual specialization. - Oecologia 140: 361-371.

McCutchan, J. H. et al. 2003. Variation in trophic shift for stable isotope ratios of carbon, nitrogen and sulfur. - Oikos 102: 378-390.

McDonald, R. A. 2002. Resource partitioning among British and Irish mustelids. - J. Anim. Ecol. 71: 185-200.

Newsome, S. D. et al. 2009. Using stable isotopes to investigate individual diet specialization in California sea otters (Enhydra lutris nereis). - Ecology 90: 961-974.

Perga, M. E. and Gerdeaux, D. 2006. Seasonal variability in the $\delta^{13} \mathrm{C}$ and $\delta^{15} \mathrm{~N}$ values of the zooplankton taxa in two alpine lakes. - Acta Oecol. 30: 69-77.

Persson, J. et al. 2010. To be or not to be what you eat: regulation of stoichiometric homeostasis among autotrophs and heterotrophs. - Oikos 119: 741-751.

Peterson, B. J. and Fry, B. 1987. Stable isotopes in ecosystem studies. - Annu. Rev. Ecol. Syst. 18: 293-320.

Phillips, D. L. and Gregg, J. W. 2001. Uncertainty in source partitioning using stable isotopes. - Oecologia 127: 171-179.

Pigeon, D. et al. 1997. Multiple modes of speciation involved in the parallel evolution of sympatric morphotypes of lake whitefish (Coregonus clupeaformis, Salmonidae). - Evolution 51: 196-205.

Robinson, B. W. et al. 1993. Ecological and morphological differentiation of pumpkinseed sunfish in lakes without bluegill sunfish. - Evol. Ecol. 7: 451-464.

Roughgarden, J. 1972. Evolution of niche width. - Am. Nat. 106: 683-718.

Svanbäck, R. and Bolnick, D. I. 2005. Intraspecific competition affects the strength of individual specialization: an optimal diet theory model. - Evol. Ecol. Res. 7: 993-1012.

Syväranta, J. and Jones, R. I. 2008. Changes in feeding niche widths of perch and roach following biomanipulation, revealed by stable isotope analysis. - Freshw. Biol. 53: 425-434.

Van Valen, L. 1965. Morphological variation and width of ecological niche. - Am. Nat. 99: 377-390.

Vander Zanden, H. B. et al. 2010. Individual specialists in a generalist population: results from a long-term stable isotope series. - Biol. Lett. 6: 711-714.

Vuorio, K. et al. 2006. Taxon-specific variation in the stable isotopic signatures $\left(\delta^{13} \mathrm{C}\right.$ and $\left.\delta^{15} \mathrm{~N}\right)$ of lake phytoplankton. - Freshw. Biol. 51: 807-822.

Willson, J. D. et al. 2010. Seasonal variation in terrestrial resource subsidies influences trophic niche width and overlap in two aquatic snake species: a stable isotope approach. - Oikos 119: 1161-1171.

Zehnder, A. and Gorham, P. R. 1960. Factors influencing the growth of Microcystis aeruginosa Kütz. Emend. Elenkin. - Can. J. Microbiol. 6: 645-660.

Zohary, T. et al. 1994. Seasonality of stable carbon isotopes within the pelagic food web of Lake Kinneret. - Limnol. Oceanogr. 39: 1030-1043. 